\title{
Apogossypolone induces apoptosis and autophagy in nasopharyngeal carcinoma cells in an in vitro and in vivo study
}

\author{
RUINIAN ZHENG ${ }^{1 *}, \mathrm{KEXU} \mathrm{CHEN}^{2 *}, \mathrm{YU} \mathrm{ZHANG}^{2}, \mathrm{JIE} \mathrm{HUANG}^{2}$, \\ FENGRONG $\mathrm{SHI}^{2}$, GANG WU ${ }^{2}$ and SENMING WANG ${ }^{2}$ \\ ${ }^{1}$ Department of Oncology, Dongguan People's Hospital, Dongguan, Guangdong 523000; ${ }^{2}$ Department of \\ Oncology, Zhujiang Hospital, Southern Medical University, Guangzhou, Guangdong 510282, P.R. China
}

Received August 7, 2015; Accepted December 16, 2016

DOI: $10.3892 / \mathrm{ol} .2017 .6176$

\begin{abstract}
Nasopharyngeal carcinoma (NPC) has a high incidence and mortality rate, particularly in Southern China. Apogossypolone (ApoG2) is a novel derivative of gossypol with antitumor activity and less toxicity. The human NPC CNE-2 cell line was studied in the in vitro model; whilst 4 week-old male nude mice (BALB/c-nu) were inoculated subcutaneously with CNE-2 cells, and xenograft tumors were studied in the in vivo model. Graded concentrations of ApoG2 were used in treatment studies. In ApoG2-treated and control in vitro and in vivo tumor cells, cell apoptosis, and autophagy were evaluated and quantified using fluorescent and transmission electron microscopy and flow cytometry. Hoechst-33258 fluorescence staining was used to evaluate apoptosis in treated and non-treated cell culture and xenograft NPC cells. Western blotting was performed on lysed tumor cells using primary antibodies to B-cell lymphoma-2 (Bcl-2), beclin-1, and $\beta$-actin, and flow cytometry results indicated cell apoptosis rates of $3.90 \pm 0.34$ and $19.52 \pm 1.18 \%$ in the control and ApoG2-treated cells, respectively ( $F=485.294$, $\mathrm{P}<0.001)$. Western blot analysis showed that ApoG2 significantly decreased expression of the $\mathrm{Bcl}-2$ protein in CNE-2 cells, when compared with control cells $(\mathrm{F}=68.909$, $\mathrm{P}=0.001)$ and flow cytometry showed cell autophagy rates of $0.92 \pm 3.10 \%$ of control cells compared with $28.24 \pm 7.35 \%$ of ApoG2-treated cells $(\mathrm{F}=31.035, \mathrm{P}=0.003)$. ApoG2 treatment significantly increased beclin-1 protein expression in CNE-2
\end{abstract}

Correspondence to: Dr Senming Wang, Department of Oncology, Zhujiang Hospital, Southern Medical University, 253 Industrial Avenue, Haizhu, Guangzhou, Guangdong 510282, P.R. China

E-mail: oncology@163.com

${ }^{*}$ Contributed equally

Abbreviations: ApoG2, apogossypolone; NPC, nasopharyngeal carcinoma

Key words: apogossypolone, apoptosis, autophagy, beclin-1, nasopharyngeal carcinoma cells $(\mathrm{F}=497.906, \mathrm{P}<0.001)$. ApoG2 treatment inhibited NPC xenograft tumor growth by $65.49 \%(\mathrm{P}<0.05)$. In conclusion, these results support a role for ApoG2 in inhibiting the growth of human NPC cells by inducing apoptosis and autophagy. Future controlled clinical studies could be planned, to define safety, efficacy and dosing regimens for ApoG2 as a potential treatment for patients with NPC.

\section{Introduction}

Nasopharyngeal carcinoma (NPC) arises from the epithelium of the nasopharyngeal mucosa. The incidence of NPC varies between countries, however among the Caucasian population, the incidence is $<1$ case per 100,000 individuals $(1,2)$. In contrast, NPC ranks among the most common of the head and neck cancers in Asia, particularly in Hong Kong and the Guangdong Province in Southern China, where the incidence is 25 cases per 100,000 individuals $(1,2)$. The majority of patients with NPC are initially diagnosed with advanced disease, resulting in a high rate of mortality $(1,2)$. Recently, healthcare providers have begun to examine the possible roles of lifestyle and genetic factors in the development of NPC. The notion that salted fish consumption is a classical risk factor of NPC in China has been denied by Lau et al (3). They also reported that vegetable consumption appears to help protect against NPC (3). Numerous therapeutic strategies have been investigated to improve the prognosis for NPC patients, including surgical techniques, chemotherapy, radiation and targeted therapies $(4,5)$. However, patients with NPC, and particularly those with relapsed NPC, continue to have a poor survival rate. Many of the current treatments for NPC have high toxicities (6). Therefore, there is an urgent need to identify novel drugs that are more effective, but less toxic for the treatment of NPC.

Apogossypolone (ApoG2) is a novel derivative of gossypol, which is a polyphenolic substance extracted from cottonseed (7). ApoG2 is an effective inhibitor of cancer cell proliferation and suppresses tumor growth by inducing apoptosis (7). Additionally, ApoG2 is less toxic to normal cells than gossypol. In laboratory and clinical studies, ApoG2 has shown potent antitumor activity for several types of malignant tumors including prostate (7), breast (8), gastric (9) and pancreatic cancer (10), myeloma (11) and chronic lymphocytic leukemia (12). Several 
studies have now demonstrated that ApoG2 induces tumor cell apoptosis by blocking the B-cell lymphoma-2 (Bcl-2) signaling pathway $(13,14)$. Bcl-2 is an anti-apoptotic protein that serves a critical role in promoting tumor cell survival and tumor growth $(15,16)$. An agent that selectively inhibits Bcl-2 expression and/or activity would be hypothesized to induce tumor cell apoptosis and inhibit tumor growth.

Given the previous studies supporting an antitumor role for ApoG2, the present study designed an in vitro and in vivo model to evaluate its effects and mechanisms in NPC, a tumor with high morbidity and mortality, particularly in Southern China.

\section{Materials and methods}

Cell lines, ApoG2, and experimental reagents. The human NPC CNE-2 cell line was obtained from the Cancer Institute of the Southern Medical University (Guangzhou, China). ApoG2 was provided by the University of Michigan (Michigan, USA). An ApoG2 stock solution was freshly prepared at a concentration of $20 \mathrm{mmol} / 1$ in $100 \%$ dimethyl sulfoxide (DMSO) on the day of the experiment, and then diluted to the specific concentrations $(0,5,10,20,40,60$ and $80 \mu \mathrm{mol} / \mathrm{l})$ required for a particular study.

Control groups in the experiments were treated with $0.1 \%$ DMSO alone. All primary antibodies used in western blot analysis were rabbit anti-human monoclonal antibodies. The anti-Bcl-2 antibody was purchased from Santa Cruz Biotechnology, Inc. (Santa Cruz, CA, USA; sc-492; dilution 1:1,000), the anti-beclin-1 antibody was purchased from Cell Signaling Technology, Inc. (Danvers, MA, USA; 3495; dilution, $1: 1,000)$ and the anti- $\beta$-actin antibody was purchased from Abmart Biomedical (Shanghai, China; P30002; dilution, 1:2,000). The secondary antibodies were biotin-labeled goat anti-rabbit antibodies purchased from Boster Biotechnology Inc. (Wuhan, China; BA1003; dilution, 1:400). A pre-stained protein ladder was purchased from Thermo Fisher Scientific, Inc., Waltham, MA, USA), and other reagents and laboratory supplies were purchased from Hyclone; GE Healthcare Life Sciences (Logan, UT, USA).

Cell culture. The human NPC CNE-2 cell line was maintained in RPMI 1640 culture medium supplemented with $10 \%$ fetal bovine serum (FBS), $100 \mathrm{U} / \mathrm{ml}$ penicillin, and $100 \mu \mathrm{g} / \mathrm{ml}$ streptomycin (Gibco; Thermo Fisher Scientific, Inc.) in a humidified incubator at $37^{\circ} \mathrm{C}$ containing $5 \% \mathrm{CO}_{2}$. Subcultures were initiated when the cell density reached $\sim 80 \%$. Cells to be harvested were trypsinized $(0.025 \%$ trypsin and $0.02 \%$ EDTA) and then washed twice with PBS.

Cell viability assay. Human NPC CNE-2 cells were seeded at a density of 5,000 cells/well in flat-bottom 96-well plates (100 $\mu \mathrm{l}$ per well). A total of $24 \mathrm{~h}$ later the cells were treated with ApoG2 at increasing concentrations $(0,5,10,20,40,60$ and $80 \mu \mathrm{mol} / \mathrm{l}$ ), and cell viability was determined after 24,48 , and $72 \mathrm{~h}$ using the Cell Counting kit-8 assay (CCK-8; Dojindo Molecular Technologies, Inc., Kumamoto, Japan) according the manufacturer's protocol. All cell viability assays were performed in triplicate.
Hoechst 33258 staining. Human NPC CNE-2 cells were seeded in 6-well plates $(50,000$ cells/well) and incubated overnight at $37^{\circ} \mathrm{C}$. Subsequently, the cells were treated with $40 \mu \mathrm{mol} / 1 \mathrm{ApoG} 2$ for $48 \mathrm{~h}$ and then fixed in $4 \%$ formaldehyde for $10 \mathrm{~min}$. The cells were then washed twice with ice-cold PBS and stained with $0.5 \mathrm{ml}$ of blue nucleic acid counterstain solution Hoechst 33258 (Sigma-Aldrich; Merck KGaA, Darmstadt, Germany) for $5 \mathrm{~min}$ at room temperature. The cells were examined under an inverted fluorescence microscope. Cells with punctate and condensed nuclei were identified as apoptotic cells.

Flow cytometry analysis of apoptotic cells. Human NPC cell line CNE-2 cells were seeded in 6-well plates and treated with $40 \mu \mathrm{mol} / 1$ ApoG2. Following a 48 -h incubation at $37^{\circ} \mathrm{C}$, cells were harvested, washed twice with ice-cold PBS, and resuspended in a binding buffer. The cells were then stained with Annexin V/propidium iodide solution (BD Biosciences, San Jose, CA, USA) at room temperature, and analyzed using a Becton Dickinson FACScan flow cytometer (BD Biosciences).

Analysis of autophagy by immunofluorescence staining. Human NPC CNE-2 cells were treated with $40 \mu \mathrm{mol} / 1$ ApoG2 for $48 \mathrm{~h}$ then washed, harvested and centrifuged at $1,500 \mathrm{xg}$ for $10 \mathrm{~min}$ at $4^{\circ} \mathrm{C}$. The pelleted cells were initially fixed for $1 \mathrm{~h}$ in a $2.5 \%$ glutaraldehyde in $0.1 \mathrm{M}$ sodium cacodylate buffer for $1 \mathrm{~h}$, and then in $1 \%$ osmium tetroxide in $0.1 \mathrm{M}$ cacodylate buffer for $1 \mathrm{~h}$. The cells were then dehydrated with ethanol and infiltrated with Araldite resin. An ultramicrotome was used to obtain specimens of 70-80 nm thickness. Finally, the cells were stained with uranyl acetate and lead citrate, and any features of autophagy were observed using transmission electron microscopy (TEM).

Autophagy detection by flow cytometry analysis. Human NPC cell line CNE-2 cells were treated with $40 \mu \mathrm{mol} / 1$ ApoG2 for $48 \mathrm{~h}$ as aforementioned and incubated with $1 \mathrm{mg} / \mathrm{l}$ acridine orange for $15 \mathrm{~min}$. The cells were then repeatedly washed with ice-cold PBS to remove residual dye, and images were captured using an inverted fluorescence microscope equipped with a $100 \mathrm{~W}$ mercury lamp (490 nm band pass blue excitation filters, a $500 \mathrm{~nm}$ dichroic mirror and a $515 \mathrm{~nm}$ long pass barrier filter). Autophagy was quantified based on the mean number of cells showing intense red staining. In total, 3 microscopic fields containing $\geq 50$ cells per field were analyzed when determining the results obtained with each set of experimental conditions. Fluorescence intensity was analyzed using the FACScan software system (BD Biosciences).

Western blot analysis. Human NPC CNE-2 cells were treated with ApoG2 at increasing concentrations (0, 5, 10, 20, 40, 60 and $80 \mu \mathrm{mol} / \mathrm{l}$ ) for $48 \mathrm{~h}$; after which they were washed twice with ice-cold PBS and lysed in $0.5 \mathrm{ml}$ lysis buffer for $20 \mathrm{~min}$ at $4^{\circ} \mathrm{C}$. Protein concentrations were determined using a BCA Protein assay kit (Thermo Fisher Scientific, Inc.). Samples with equivalent amounts of total protein were loaded and separated by $12 \%$ SDS-PAGE, and then transferred to a polyvinylidene difluoride membrane. Immunoblotting was performed using 
primary antibodies against $\mathrm{Bcl}-2$, beclin- 1 , and $\beta$-actin, and a horseradish peroxidase-conjugated anti-IgG as the secondary antibody. The blots were developed using ECL chemiluminescent reagent (Cell Signaling Technology, Inc.).

In vivo tumor model in BALB/C-nu mice. A total of 30 male nude mice (BALB/c-nu) of 4 weeks old, weighting 16-19 g, were purchased from the Provincial Animal Center (Guangdong, China), and maintained under a fixed $12 \mathrm{~h}$ light/dark photoperiod (lights on from 7:00 to 19:00) with food and water available ad libitum. They were raised individually housed in a stainless steel cage in a room maintained at $25 \pm 1^{\circ} \mathrm{C}$ with $70 \pm 4 \%$ relative humidity. All animal studies were performed in accordance with guidelines provided in the Guide for the Care and Use of Laboratory Animals (14).

The study protocols were approved by the Animal Investigation Committee of Sun Yat-Sen University (Guangzhou, China). The human NPC CNE-2 cells suspended in serum-free culture medium were inoculated subcutaneously into the flank region of BALB/c-nu mice $\left(5 \times 10^{6}\right.$ cells/mouse). When the tumor size reached $\sim 4 \mathrm{~mm}$ in diameter, the mice were randomly assigned to subgroups consisting of a control group and an ApoG2-treatment group. All pharmacologic agents were administered by intraperitoneal injection at a dose of $120 \mathrm{mg} / \mathrm{kg}$ every other day for 3 weeks. The weight of each mouse and the tumor volume was monitored every other day. Tumor measurements were obtained using vernier calipers, and tumor volumes were calculated as $\mathrm{AxBxB} / 2$; where $A$ and $B$ represent tumor length and width, respectively. At 2 weeks post-inoculation, the mice were anesthetized using $10 \%$ chloral hydrate (47335; Sigma-Aldrich; Merck KGaA injected intraperitoneally $(300 \mathrm{mg} / \mathrm{kg})$. Immediately after, they were euthanized by dislocated cervical vertebra and their tumor xenografts were removed. The percent inhibition of tumor growth was calculated as (1-T/C)x100\%; where T and C represent the average tumor weight in the ApoG2 and control group, respectively.

Statistical analysis. All statistical analysis was performed using SPSS statistics software for Windows, version 13.0 (SPSS Inc., Chicago, IL, USA). Data was calculated as a percentage obtained from at least 3 duplicate experiments and expressed as the mean \pm standard deviation. Statistical differences between mean values were analyzed using the Student's t-test or one-way analysis of variance. $\mathrm{P}<0.05$ was considered to indicate a statistically significant difference.

\section{Results}

ApoG2 decreased CNE-2 cell viability. The CCK-8 assay results indicated that ApoG2 significantly decreased the viability of the human NPC cell line CNE-2 cells in a time and dose-dependent manner $\left(\mathrm{F}_{\text {time }}=2041.671, \mathrm{P}_{\text {time }}<0.001 ; \mathrm{F}_{\text {concentra- }}\right.$ tion $=1819.354, \mathrm{P}_{\text {concentration }}<0.001 ;$ Fig. 1). The results also suggest an interaction between ApoG2 concentration and exposure time $(\mathrm{F}=202.540, \mathrm{P}<0.001)$. The $50 \%$ inhibitory concentration $\left(\mathrm{IC}_{50}\right)$ for ApoG2 after a $72 \mathrm{~h}$ exposure was $23.61 \mu \mathrm{mol} / 1$.

ApoG2 induced apoptosis in CNE-2 cells. Hoechst-33258 nucleic acid staining (blue) demonstrated apoptosis in CNE-2

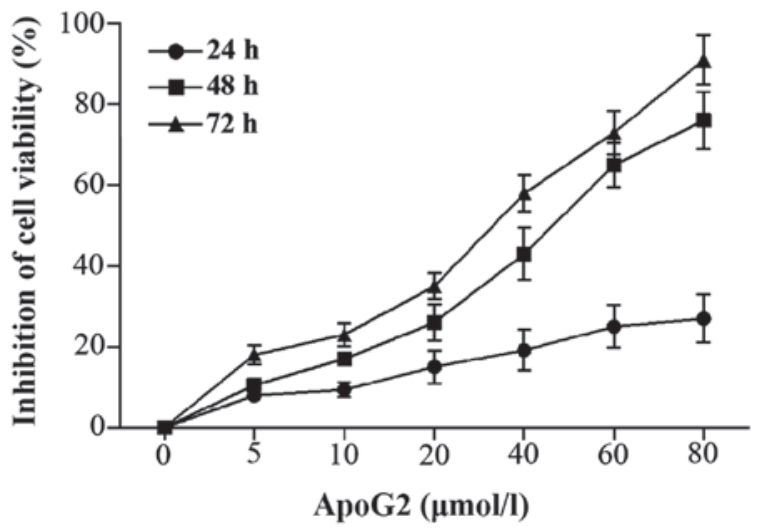

Figure 1. ApoG2 treatment reduced the viability of human nasopharyngeal carcinoma CNE-2 cells in a dose and time-dependent manner. Data are presented as the mean \pm standard deviation $(n=3)$. ApoG2, apogossypolone.

cells following incubation with ApoG2. Cell nuclear pyknosis, chromosome fragmentation, chromatin condensation, the formation of apoptotic bodies, and other apoptotic features were observed in ApoG2-treated cells but not in control cells (Fig. 2A). Flow cytometry results indicated apoptosis rates of $3.90 \pm 0.34$ and $19.52 \pm 1.18 \%$ in the control and ApoG2 treated cells, respectively, and this difference was statistically significant $(\mathrm{F}=485.294, \mathrm{P}<0.001$; Fig. 2B). Western blot analysis showed that ApoG2 significantly decreased expression of Bcl-2 protein in CNE-2 cells, when compared with expression in control cells $(\mathrm{F}=68.909, \mathrm{P}=0.001$; Fig. $2 \mathrm{C}$ and $\mathrm{D})$.

ApoG2 induced autophagy in CNE-2 cells. Control cells stained with acridine orange showed a cell nucleus and cytoplasm that appeared bright green. In contrast, ApoG2-treated cells showed bright red fragments of cytoplasm and nucleus contained within acidic autophagosomes (Fig. 3A). Increased numbers of large vacuoles and double-layered membrane structures were observed by TEM in ApoG2-treated cells, but not in control cells (Fig. 3B). Flow cytometry results indicated that $0.92 \pm 3.10 \%$ of control cells exhibited fluorescence compared with $28.24 \pm 7.35 \%$ of ApoG2-treated cells $(\mathrm{F}=31.035, \mathrm{P}=0.003$; Fig. 3C). Additionally, ApoG2 treatment significantly increased beclin-1 protein expression in CNE-2 cells $(\mathrm{F}=497.906, \mathrm{P}<0.001$; Fig. 3D and $\mathrm{E})$.

ApoG2 suppressed tumor growth in the BALB/c-nu mice. The in vivo study of subcutaneously grafted CNE-2 human NPC cells in BALB/c-nu mice showed that ApoG2 treatment inhibited tumor growth by $65.49 \%(\mathrm{P}<0.05)$. The mice showed no adverse reactions to treatment throughout the study. Based on these results, the ApoG2 treatment was demonstrated to suppress tumor growth in this in vivo mouse model (Fig. 4).

\section{Discussion}

NPC is associated with a high mortality rate worldwide, but is a particularly prevalent primary malignancy in China (17). $\mathrm{Xu}$ et al (18) have reported that the incidence of mortality due to NPC in China is $1.99 / 100,000$ individuals, and mortality in males with NPC is greater than in females at 2.81/100,000 


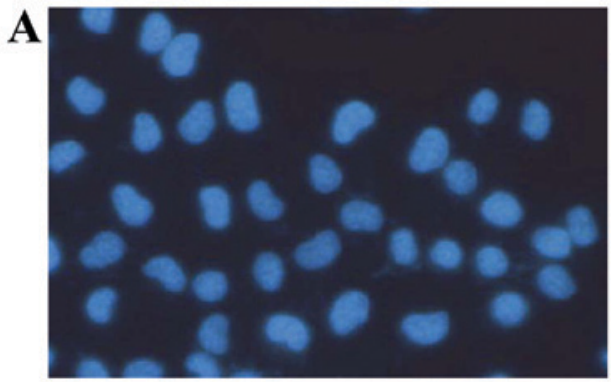

Control

B

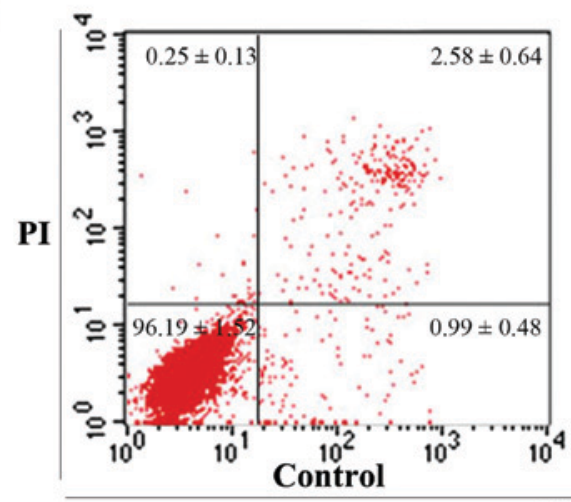

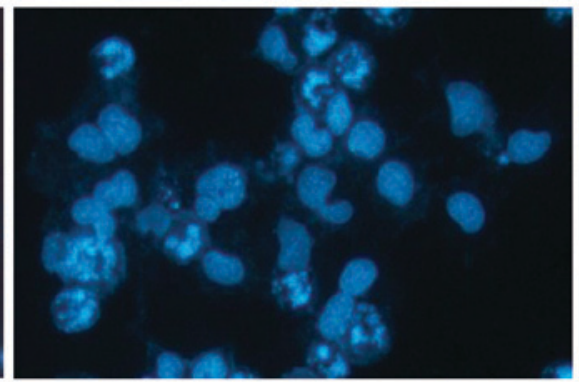

ApoG2

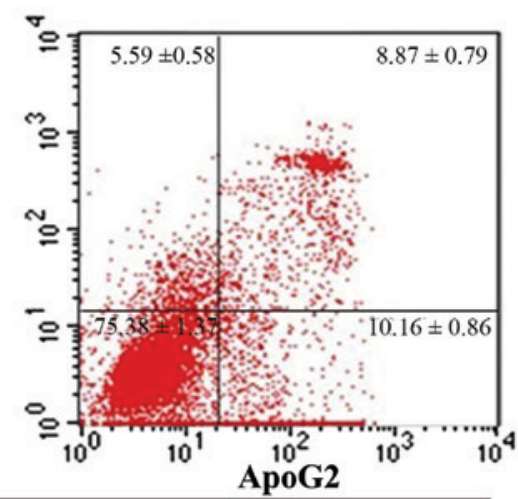

FITC
C

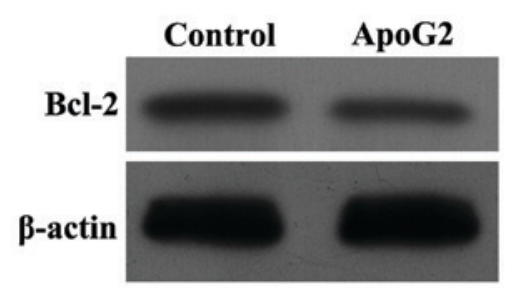

D

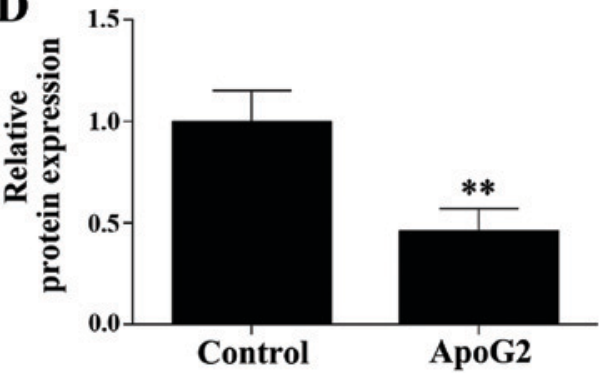

Figure 2. ApoG2 treatment-induced apoptosis in human nasopharyngeal carcinoma CNE-2 cells. (A) Hoechst staining (blue) and (B) flow cytometry analysis showed that ApoG2 induced CNE-2 cell apoptosis. (C) Western blot analysis indicated that ApoG2 inhibited Bcl-2 expression in CNE-2 cells. (D) Quantification of Bcl-2 expression levels in each group is presented in bar graphs as the fold-increase. ${ }^{* *} \mathrm{P}<0.01$ vs. the control group, data are presented as the mean \pm standard deviation. ApoG2, apogossypolone; PI, propidium iodide; FITC, fluorescein isothiocyanate; Bcl-2, B-cell lymphoma-2.

vs. 1.14/100,000, respectively (19). Chemoradiotherapy remains the standard treatment for locally-advanced and lymph node-positive NPC, and platinum-based chemotherapy treatment regimens have recently demonstrated superiority (17). Previous studies have demonstrated that lifestyle changes may to help to prevent NPC, and that early detection can improve patient survival $(17,19)$. Despite the use of conventional chemotherapy, radiation and biological therapy and preventive strategies such lifestyle changes, there remains a requirement to identify safe and effective treatments for NPC.

The present study used in vitro and in vivo models of NPC to demonstrate that ApoG2 is capable of decreasing NPC tumor cell viability and suppressing tumor growth. Apoptosis, or programmed cell death, assists in eliminating unhealthy cells that are generated in physiological or pathological conditions, oncogene activation, hypoxia, or during chemotherapy or radiation $(18,20)$. As an important anti-apoptosis protein, Bcl-2 serves a critical role in regulating cell survival and modulates the activity of tumor growth-associated molecules $(21,22)$. Several studies have demonstrated that overexpression of Bcl-2 enhances tumor cell growth whilst silencing of Bcl-2 with small interfering RNA significantly inhibited the growth of tumor cells (23-25). It is also known that chemotherapy resistance in patients with NPC correlates with overexpression of Bcl-2 while inhibition of Bcl-2 enhances sensitivity to chemotherapy $(26,27)$. As a result, targeted downregulation of Bcl-2 expression is considered an effective strategy for sensitizing tumor cells to chemotherapy.

Autophagy has been widely accepted as a type of programmed cell death. However, it remains unclear whether autophagy is beneficial or detrimental for tumor growth. Autophagy serves to eliminate superfluous or damaged organelles and initiates the formation of multiple double-membrane vacuoles that fuse with lysosomes to form intracellular bodies termed 'autophagosomes' $(28,29)$. Certain external factors such as nutrient deprivation and specific medications can induce autophagy and lead to decreased cell viability. On the basis of these published findings on the importance of apoptosis and 
A
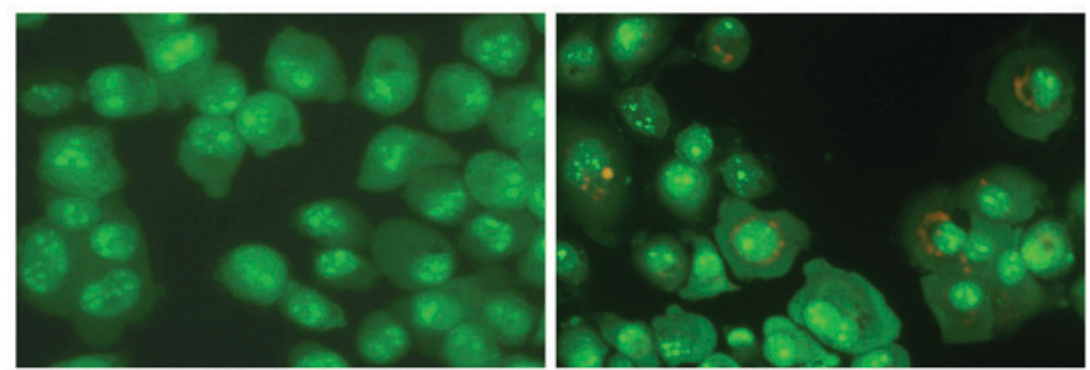

Control

ApoG2

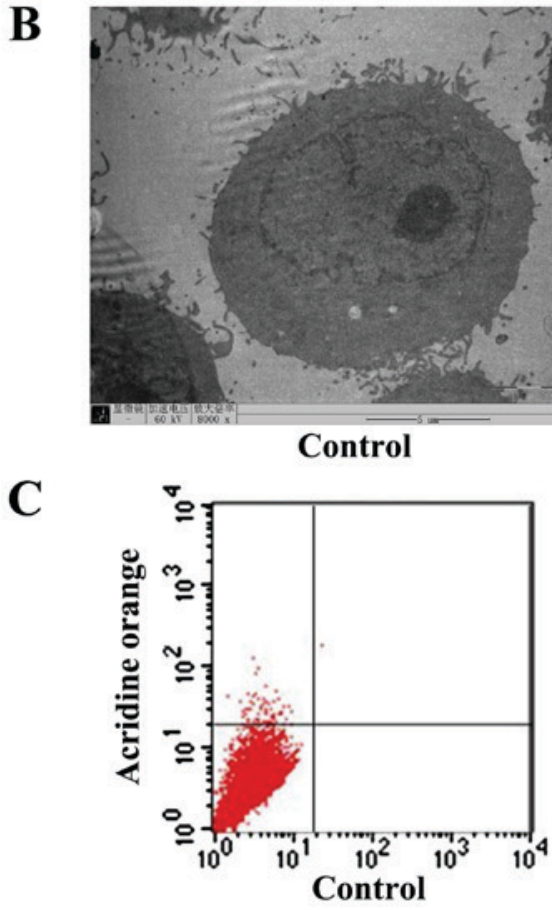

D
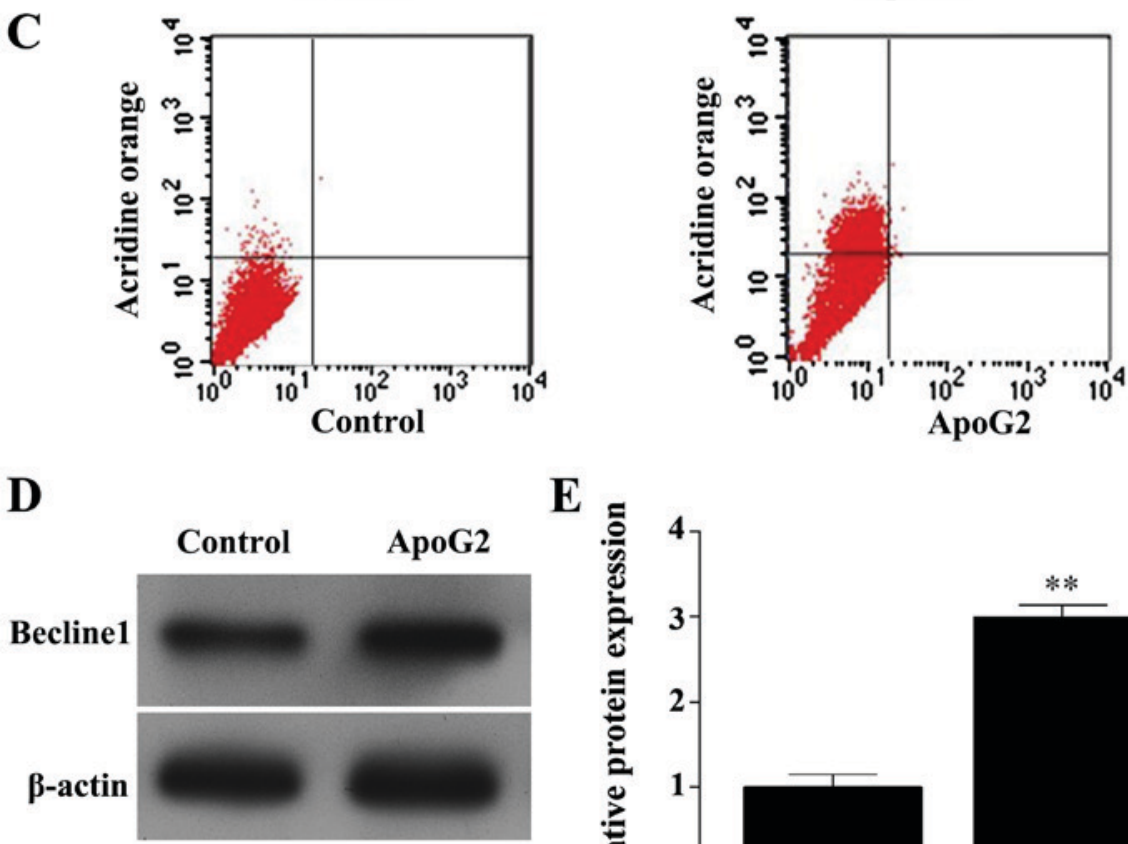

$\mathbf{E}$

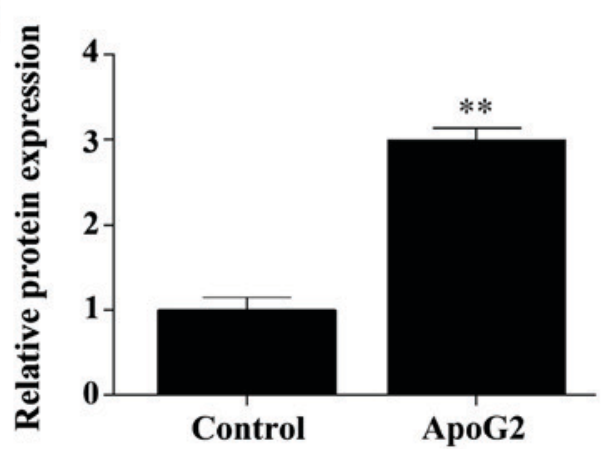

Figure 3. ApoG2 treatment-induced autophagy in CNE-2 cells. (A) Fluorescence staining, (B) transmission electron microscopy imaging, and (C) flow cytometry analysis showed ApoG2-induced autophagy in CNE-2 cells. (D) Western blot analysis showed ApoG2-induced beclin-1 expression in CNE-2 cells. (E) Quantification of beclin-1 expression levels in each group, presented in bar graphs as the fold-increase. ${ }^{* *} \mathrm{P}<0.01$ vs. the control group, data are presented as the mean \pm standard deviation $(n=3)$. ApoG2, apogossypolone.

autophagy in tumor biology, the components of the present study were planned.

The results of the current study indicated that ApoG2 significantly decreased Bcl-2 expression, leading to human NPC CNE-2 cell apoptosis. Immunofluorescence assays, flow cytometry analysis, and TEM imaging suggested that ApoG2 treatment significantly decreased autophagy in CNE-2 cells, which may be a mechanism for the observed ApoG2-induced decrease in CNE-2 cell viability. The present study additionally identified that ApoG2 treatment significantly increased expression of beclin-1 protein, which has been reported as a marker of autophagy $(30,31)$. The results of the current study are supported by several other studies that have investigated ApoG2 in NPC using either in vitro or xenograft models (32-36).

Data from the present study support a role for ApoG2 in the regulation of Bcl-2 and beclin-1 expression in the NPC CNE-2 cell line, inducing apoptosis and autophagy, which modulate tumor cell viability and tumor growth. Based on these observations, the present study suggests that additional 
A

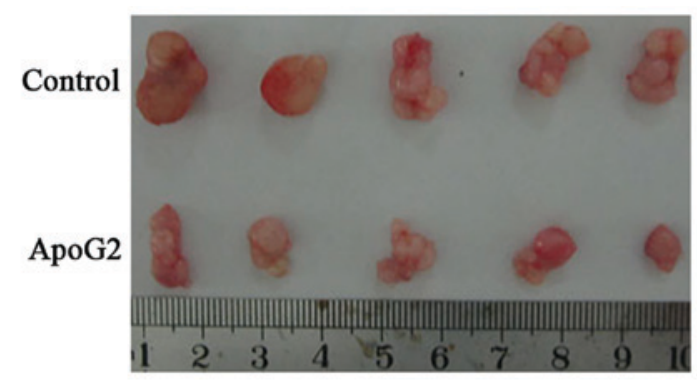

B
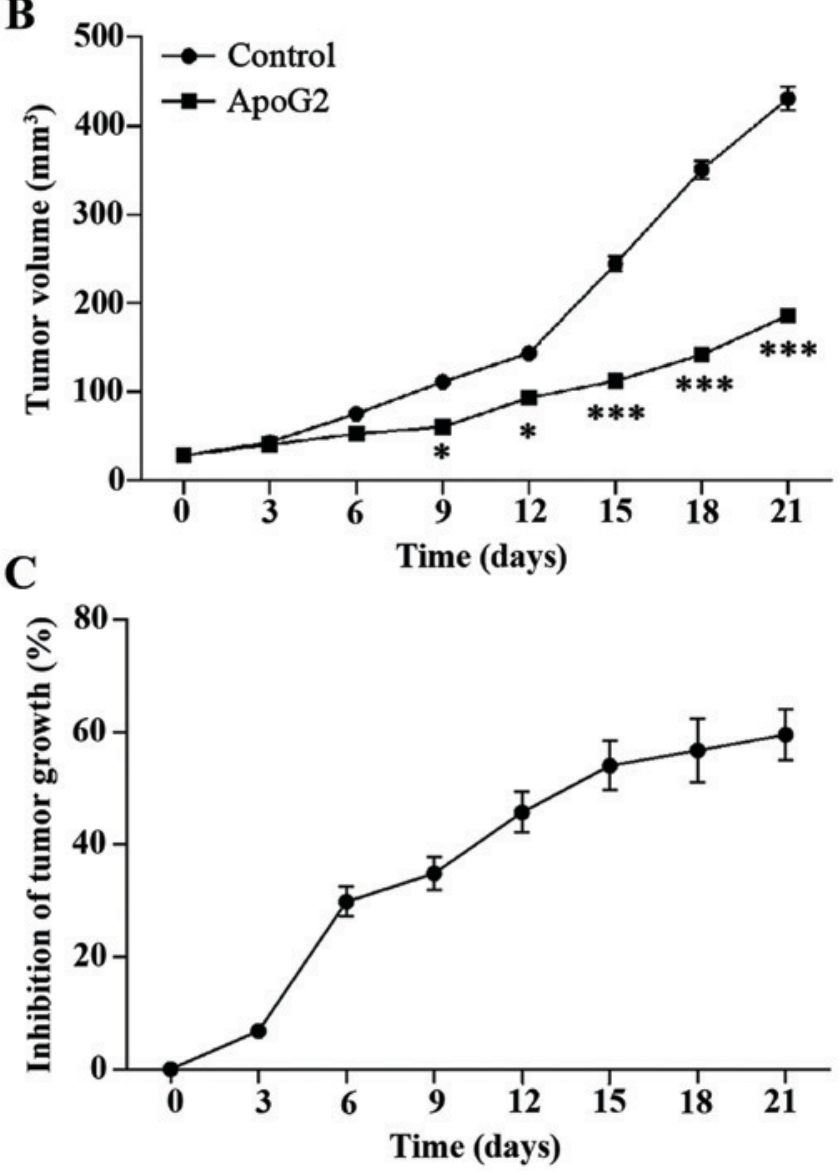

Figure 4. ApoG2-inhibited tumor growth in a BALB/c-nu mouse model of NPC. (A) Image showing human NPC tumor xenografts removed from the mice. (B) The tumor growth curves in each group. ${ }^{\mathrm{P}}<0.05,{ }^{* * * *} \mathrm{P}<0.001$ vs. the control group in $9,12,15,18,20$ days, respectively. (C) The rate of tumor growth inhibition. Data are presented as the mean \pm standard deviation $(n=3)$. ApoG2, apogossypolone; NPC, nasopharyngeal carcinoma.

larger studies on the role of ApoG2 should be performed to investigate its potential role as an antitumor agent for use in the treatment of patients with NPC.

The limitations of the present study include the small study size and the use of an NPC cell line. Cell lines may not reflect the behavior of human tumors arising in the nasopharynx that can be heterogeneous and exhibit low-grade to high-grade differentiation and behavior. In the in vivo mouse model, the 'tumors' evaluated were subcutaneously implanted and would not be expected to behave in the same way as tumors arising in the nasopharynx.

There are remaining questions to be answered regarding the mechanism of action of ApoG2 in NPC prior to conducting clinical safety and efficacy studies in humans. It is possible, for example, that autophagy and the promotion of apoptosis may promote tumor cell survival in the clinical situation. The appropriate clinical dose of ApoG2 additionally remains to be determined for patients with NPC. There is the possibility of cross-talk between apoptosis and autophagy, and the mechanism by which ApoG2 induces tumor cell autophagy remains to be studied. In addition, due to the fact that anti-Bcl-2 therapy has been reported to sensitize tumor cells to chemotherapy and radiation therapy, the present study proposed to test the effects of combined ApoG2 therapy with chemotherapy and/radiotherapy on the development of NPC in the study models.

In conclusion, these results support a role for ApoG2 in inhibiting the growth of human NPC cells by inducing apoptosis and autophagy. Additional controlled clinical studies could be planned, to define safety, efficacy and dosing regimens for ApoG2 as a potential treatment for patients with NPC.

\section{Acknowledgements}

The present study was supported by Science and Technology projects in Guangzhou, China (grant no. 2011y2-00019-3). 


\section{References}

1. Wang Y, Zhang Y and Ma S: Racial differences in nasopharyngeal carcinoma in the United States. Cancer Epidemiol 37: 793-802, 2013

2. Kimura Y, Suzuki D, Tokunaga T, Takabayashi T, Yamada T, Wakisaka N, Yoshizaki T, Murata H, Miwa K, Shoujaku H, et al: Epidemiological analysis of nasopharyngeal carcinoma in the central region of Japan during the period from 1996 to 2005. Auris Nasus Larynx 38: 244-249, 2011.

3. Lau HY, Leung CM, Chan YH, Lee AW, Kwong DL, Lung ML and Lam TH: Secular trends of salted fish consumption and nasopharyngeal carcinoma: A multi-jurisdiction ecological study in 8 regions from 3 continents. BMC Cancer 13: 298, 2013.

4. Xu T, Shen C, Ou X, He X, Ying H and Hu C: The role of adjuvant chemotherapy in nasopharyngeal carcinoma with bulky neck lymph nodes in the era of IMRT. Oncotarget 7: 21013-21022, 2016

5. Xu T, Liu Y, Dou S, Li F, Guan X and Zhu G: Weekly cetuximab concurrent with IMRT aggravated radiation-induced oral mucositis in locally advanced nasopharyngeal carcinoma: Results of a randomized phase II study. 51: 875-879, 2015.

6. Li H, Wang DL, Liu XW, Chen MY, Mo YX, Geng ZJ and Xie CM: MRI signal changes in the skull base bone after endoscopic nasopharyngectomy for recurrent NPC: A serial study of 9 patients. Eur J Radiol 82: 309-315, 2013.

7. Zhan YH, Huang XF, Hu XB, An QX, Liu ZX and Zhang XQ Growth inhibition and apoptosis induction of human umbilical vein endothelial cells by apogossypolone. Asian Pac J Cancer Prev 14: 1791-1795, 2013.

8. Niu X, Li S, Wei F, Huang J, Wu G, Xu L, Xu D and Wang S: Apogossypolone induces autophagy and apoptosis in breas cancer MCF-7 cells in vitro and in vivo. Breast Cancer 21 : 223-230, 2014.

9. Xin J,Zhan YH, Xia LM, Zhu HW, Nie YZ, Liang JM and Tian J: ApoG2 as the most potent gossypol derivatives inhibits cell growth and induces apoptosis on gastric cancer cells. Biomed Pharmacother 67: 88-95, 2013.

10. Banerjee S, Choi M, Aboukameel A, Wang Z, Mohammad M, Chen J, Yang D, Sarkar FH and Mohammad RM: Preclinical studies of apogossypolone, a novel pan inhibitor of bcl-2 and mcl-1, synergistically potentiates cytotoxic effect of gemcitabine in pancreatic cancer cells. Pancreas 39: 323-331, 2010.

11. Lin J, Wu YJ, Yang DJ and Zhao YQ: Effect of apogossypolone on induction apoptosis in multiple myeloma cells and its mechanisms. Zhongguo Shi Yan Xue Ye Xue Za Zhi 17: 92-98, 2009 (In Chinese).

12. Balakrishnan K, Aggarwal S, Wierda W and Gandhi V: Bax and $\mathrm{Bak}$ are required for apogossypolone, a $\mathrm{BH} 3$-mimetic, induced apoptosis in chronic lymphocytic leukemia cells. Leuk Lymphoma 54: 1097-1100, 2013.

13. Cheng P, Ni Z, Dai X, Wang B, Ding W, Rae Smith A, Xu L, Wu D, $\mathrm{He} \mathrm{F}$ and Lian J: The novel BH-3 mimetic apogossypolone induces Beclin-1- and ROS-mediated autophagy in human hepatocellular carcinoma [corrected] cells. Cell Death Dis 4: e489, 2013.

14. Sun J, Li ZM, Hu ZY, Lin XB, Zhou NN, Xian LJ, Yang DJ and Jiang WQ: ApoG2 inhibits antiapoptotic Bcl-2 family proteins and induces mitochondria-dependent apoptosis in human lymphoma U937 cells. Anticancer Drugs 19: 967-974, 2008.

15. Hockenbery DM: Bcl-2 in cancer, development and apoptosis J Cell Sci Suppl 18: 51-55, 1994

16. Raffo AJ, Perlman H, Chen MW, Day ML, Streitman JS and Buttyan R: Overexpression of bcl-2 protects prostate cancer cells from apoptosis in vitro and confers resistance to androgen depletion in vivo. Cancer Res 55: 4438-4445, 1995.

17. National Research Council (US) Committee for the Update of the Guide for the Care and Use of Laboratory Animals. 'Guide for the Care and Use of Laboratory Animals.' Guide for the Care \& Use of Laboratory Animals 103: 1072-1073, 2011.

18. Xu ZJ, Zheng RS, Zhang SW, Zou XN and Chen WQ: Nasopharyngeal carcinoma incidence and mortality in China in 2009. Chin J Cancer 32: 453-460, 2013.
19. Mesia R, Pastor M, Grau JJ and del Barco E; SEOM: SEOM clinical guidelines for the treatment of nasopharyngeal carcinoma 2013. Clin Transl Oncol 15: 1025-1029, 2013.

20. Piro LD: Apoptosis, Bcl-2 antisense, and cancer therapy. Oncology (Williston Park) 18 (13 Suppl 10): S5-S10, 2004.

21. Kontos CK, Christodoulou MI and Scorilas A: Apoptosis-related BCL2-family members: Key players in chemotherapy. Anticancer Agents Med Chem 14: 353-374, 2014.

22. Kaneko T, Zhang Z, Mantellini MG, Karl E, Zeitlin B, Verhaegen M, Soengas MS, Lingen M, Strieter RM, Nunez G and Nör JE: Bcl-2 orchestrates a cross-talk between endothelial and tumor cells that promotes tumor growth. Cancer Res 67: 9685-9693, 2007.

23. Tucker CA, Kapanen AI, Chikh G, Hoffman BG, Kyle AH, Wilson IM, Masin D, Gascoyne RD, Bally M and Klasa RJ: Silencing Bcl-2 in models of mantle cell lymphoma is associated with decreases in cyclin D1, nuclear factor-kappaB, p53, bax, and p27 levels. Mol Cancer Ther 7: 749-758, 2008.

24. Tekedereli I, Alpay SN, Akar U, Yuka E, Ayugo-Rodriguez C, Han HD, Sood AK, Lopez-Berestein G and Ozpolat B: Therapeutic silencing of Bcl-2 by systemically administered siRNA nanotherapeutics inhibits tumor growth by autophagy and apoptosis and enhances the efficacy of chemotherapy in orthotopic xenograft models of ER (-) and ER (+) breast cancer. Mol Ther Nucleic Acids 2: e121, 2013

25. Du P, Cao H, Wu HR, Zhu BS, Wang HW, Gu CW, Xing CG and Chen W: Blocking Bcl-2 leads to autophagy activation and cell death of the HEPG2 liver cancer cell line. Asian Pac J Cancer Prev 14: 5849-5854, 2013

26. Akar U, Chaves-Reyez A, Barria M, Tari A, Sanguino A, Kondo Y, Kondo S, Arun B, Lopez-Berestein G and Ozpolat B: Silencing of Bcl-2 expression by small interfering RNA induces autophagic cell death in MCF-7 breast cancer cells. Autophagy 4: 669-679, 2008

27. Paoluzzi L, Gonen M, Gardner JR, Mastrella J, Yang D, Holmlund J, Sorenen M, Leopold L, Manova K, Marcucci G, et al: Targeting Bcl-2 family members with the BH3 mimetic AT-101 markedly enhances the therapeutic effects of chemotherapeutic agents in in vitro and in vivo models of B-cell lymphoma. Blood 111: 5350-5358, 2008.

28. Lieber J, Kirchner B, Eicher C, Warman SW, Seitz G, Fuchs J and Armeanu-Ebinger S: Inhibition of Bcl-2 and Bcl-X enhances chemotherapy sensitivity in hepatoblastoma cells. Pediatr Blood Cancer 55: 1089-1095, 2010.

29. Lamb CA, Yoshimori T and Tooze SA: The autophagosome: Origins unknown, biogenesis complex. Nat Rev Mol Cell Biol 14: 759-774, 2013.

30. Yang Z and Klionsky DJ: Eaten alive: A history of macroautophagy. Nat Cell Biol 12: 814-822, 2010.

31. Xu HD, Wu D, Gu JH, Ge JB, Wu JC, Han R, Liang ZQ and Qin ZH: The pro-survival role of autophagy depends on Bcl-2 under nutrition stress conditions. PLoS One 8: e63232, 2013.

32. Tian S, Lin J, Jun Zhou J, Wang X, Li Y, Ren X, Yu W, Zhong W, Xiao J, Sheng F et al: Beclin 1-independent autophagy induced by a $\mathrm{Bcl}-\mathrm{XL} / \mathrm{Bcl}-2$ targeting compound, Z18. Autophagy 6 : $1032-1041,2010$

33. He JH, Liao XL, Wang W, Li DD, Chen WD, Deng R, Yang D, Han ZP, Jiang JW and Zhu XF: Apogossypolone, a small-molecule inhibitor of Bcl-2, induces radiosensitization of nasopharyngeal carcinoma cells by stimulating autophagy. Int J Oncol 45: 1099-1108, 2014

34. Hu ZY, Wang J, Cheng G, Zhu XF, Huang P, Yang D and Zeng YX: Apogossypolone targets mitochondria and light enhances its anticancer activity by stimulating generation of singlet oxygen and reactive oxygen species. Chin J Cancer 30: 41-53, 2011.

35. Hu ZY, Sun J, Zhu XF, Yang D and Zeng YX: ApoG2 induces cell cycle arrest of nasopharyngeal carcinoma cells by suppressing the c-Myc signaling pathway. J Transl Med 7: 74, 2009.

36. Hu ZY, Zhu XF, Zhong ZD, Sun J, Wang J, Yang D and Zeng YX: ApoG2, a novel inhibitor of antiapoptotic Bcl-2 family proteins, induces apoptosis and suppresses tumor growth in nasopharyngeal carcinoma xenografts. Int J Cancer 123: 2418-2429. 2008. 